\title{
Design Web-based Services to Enhance Telemedicine Database Management System using Integrated-Fragmentation-Clustering- Allocation
}

\author{
Mashette Padma ${ }^{1}$, Sapkal Trupti $^{1}$, Khade Sushama $^{1}$, Priyam Aditi $^{1}$, Rohit Bamane $^{1}$ \\ Department of Computer Engineering Dr. D. Y. Patil Institute of Engineering and Technology, University of Pune ${ }^{1}$
}

\begin{abstract}
This paper will focus on the telecommunication between the patient and doctor. Telemedicine is a exchanging of information from one location to another location via electronic communication. The main purpose of the project is to transfer medical services to the patient, rather than the transport of the patient to the medical services. The main achievement by using this application it gives services which is easy to handle, faster and less expensive. This Application used three techniques data Fragmentation, website Clustering and data distribution. It reduce the communication cost between websites during the processing of application. We propose a doctor patient interaction application where admin will add the doctors (admin must have a portal to do so). Doctors can view the appointments for the day also the doctor can prescribe the medicine through web application. Patients' details can be added using web portal and also using web application. Admin can view patient details, appointments, doctor's details and can also upload patients test reports. It's a complete doctor patient interaction application which is being automated for the ease of use. The overall result shows that these combined three approach which improves the performance of web database systems and outperforms its correlated work. An approach to manage multimedia medical database in telemedicine system is produced.
\end{abstract}

Keywords: Telemedicine, Web Telemedicine Database Systems, Data Fragmentation, Website clustering, and Data Allocation.

\section{INTRODUCTION}

Now days, a rapid growth of the real world software application. The web plays an important role in the telemedicine. Telemedicine is the interaction between patient and doctor via electronic communication, providing a medical services over the treatment in the text, Image format or any text file. The issue like distance, time, travel overcome by this application the transport cost, travelling time. We propose a doctor patient interaction application where admin will add the doctors (admin must have a portal to do so). Doctors can view the appointments for the day, also the doctor can prescribe the medicine through web application. Patients' details can be added using web portal and also using web application. Admin Panel Web part (Receptionist) can view patient details, appointments, doctor's details and can also upload patients test reports. It's a complete doctor patient interaction application which is being automated for the ease of use.

The web play a very important role in enabling healthcare services like telemedicine. It is easy and global access to patients' data without having to communicate with them in person $\mathrm{n}$ it provides fast channels to communicate specialists in emergency situations. There are different kinds of information such as ECG, temperature, asthma, allergies, liver and heart rate need to be accessed by various client devices in heterogeneous communications environments. Many techniques have been proposed in order to improve telemedicine database performance, optimize medical data given out, and maintain medical data in multiple way as given above.

To improve the performance of medical distributed database systems, we include data fragmentation, websites clustering, and data distribution computing services together in a new web telemedicine database system approach. This new method plans to decrease data communication, increase system throughput, reliability and availability. These technique ensure that high performance for such systems can be achieved by improving at least one of the database web management services such as database fragmentation, website clustering, data distribution, data allocation, distributed caching, and database scalability. These methods supposed that high performance for such systems can be achieved by improving at least one of web database management services such as database fragmentation, data distribution, websites clustering, distributed caching and scalability.

Designing and developing fast, efficient, and reliable included techniques that can handle large number of medical transactions on huge number of web healthcare sites in close optimal polynomial time are key challenges in area of 
web-based telemedicine database system. Three approaches such as data fragmentation, websites clustering, and data allocation are the main components of the web-based telemedicine database system that continue to create great research challenges as their current close optimal solutions are all NP-Complete. The advantages using web portal to minimize time and distance gap between doctors and patients and to provide fast and adequate medical services. Through the connection between web portal terminals and specific service, both doctors and patients are able to obtain required data to achieve a better interaction.

To solve the problem, we develop a database management methodology by which we can get information about medical service that can be organized based upon the patient's complaint as well as his/her medical historical data. We also provide a unified interface for manipulating and accessing the different types of medical information as we know. The management of medical database and the user interface has been implemented in it. Due to insufficiency of medical resources and lack of opportunities for continuing education, physician may be reluctant to serve in rural areas or geographically isolated regions around us. So people who live in these rural sector may receive lower effectively medical care than those who live in other area like urban. Therefore it is very important to develop a telemedicine database management system for improving the quality of medical services and providing more educational opportunities to physicians in rural areas.

\section{OBJECTIVE}

1. It provide large data of medical data records.

2 .It provide accurate decision support system.

3. It provide treatment to the patient at anytime, anywhere.

4. The benefits of the applications are given as follows:

- Transportation cost saving.

- Time saving.

- Medical consultation delivery.

\section{LITERATURE SURVEY}

1. D. A. Vidhate1, Ige Pranita2, Kothari Pooja3, Kshatriya Pooja4 "Implementing Web-Based Computing Services To Improve Performance And Assist Telemedicine Database Management System”2016.

Database management system with application in real time telemedicine database management systems to maximize care admissions and minimize care difficulties issues. There are many issues such as distance, travel, and time complexity. Three-fold approach on the basis of database fragmentation, database website clustering and intelligent data distribution (allocation). This approach decreases the amount of the data transmission between many website during applications execution to achieve cost efficient communication between website during applications processing. This improves applications response time, reliability, flexibility and throughput. The implemented system will validate by checking the impact of computing services' technology on various different kinds of performance characteristics such as communications cost, response time, and throughput. The overall results show that this combined approach specifically improves the performance of web database system.

2. Hafez Fouad Microelectronics Dept., Electronics Research Institute, Cairo, Egypt "Web-Based Database Management System for the telemedicine System" by,2014.

The aim of the this paper is to transfer of the medical care services to the patient rather than the transport of the patient to the medical services providers. This is achieved by using web-based applications including Modern Medical Informatics Services which is very easy, faster and less expensive. The required system implements the suitable informatics and electronics solution efficiently for the Telemedicine system care. We will proposed an approach to manage different multimedia medical system database in the telemedicine database system. In order to be effectively and efficiently identify, manage, search, and display database information. We define an information package for both doctor and patient. There is data set of their medical information from each visit. The methodology for approaches various types of medical records will also be provided by medical system in telemedicine management system. Also we will design two web-based interfaces such as high-quality data and display for many medical service provides.

3. Hafez Fouad Microelectronics Dept., Electronics Research Institute, Cairo, Egypt, hafez@eri.sci.eg,” Web-based Medical Informatics to Support Telemedicine System”,2014.

The remarkable growing of telemedicine implementation around the world allow users to enjoy better health services by increasing attention, patient comfort and opportunities to achieve good diagnosis among other advantages. This is achieved by using web-based applications which including various Modern Medical Informatics Services which is very easy, faster and less expensive. This system will be efficient by finding the suitable informatics and electronics 
solution for the Tele-medicine management healthcare system. The telemedicine system is proposed by approaching to manage multimedia medical database system. In order to identify, manage, search, and display patient information more efficiently. We also define a doctor and patient information package. This is data set of a doctor's information and patient's medical information from each visit. We also provide the methodology for approaches various types of patient medical records. We will design two types of user interfaces such as high-quality data display and web-based interface for different kinds of medical services provide.

4. Lepakshi Goud, "Achieving Availability, Elasticity and Reliability of the Data Access in Cloud Computing," Int'l J. Advanced Eng. Sciences and Technologies, vol. 5, no. 2, pp. 150-155, 2011.

Advantage:- High traffic to multiple sites, and some delays are tolerated.

Disadvantage:- Poor due to fragments (Packets) redundancy and Restrictions on the final fragments (Packets).

5. S. Jagannatha, M. Mrunalini, T. Kumar, and K. Kanth, "Modeling of Mixed Fragmentation in Distributed Database Using UML 2.0," IPCSIT, V. 2, pp. 190-194, 2011.

Advantage:- Easy for each site until there is need to share data across sites.

Disadvantage:- Poor due to fault tolerant risk in it.

\section{RELATED WORK}

Many Research work have attempt to improve performance of a collection of multiple interconnected databases management system. This work mostly finds out fragmentation, data allocation \& sometimes website clustering problems. In this section we allow solution for this problem, discuss and estimate their contribution with proposed system. Following is the three approaches given below.

\section{A. Data Fragmentation:}

Data fragmentation is developed by splitting telemedicine database relations into small disjoint fragments. It generates minimum number of disjoint fragments that would be allocated to the web servers in the data distribution phase. Data fragmentation reduces the data transferred \& accessed through different websites \& accordingly reduces the communication cost. It is important to divide the relation into smaller data fragments \& consider it for distribution over the network sites.

Database partitioning techniques aim at improving database systems throughput by decreasing the amount of irrelevant data fragments to be accessed and transferred among different websites. Data fragmentation increases some difficulties; particularly when web telemedicine database applications had contradictory requirements that prevent breakdown of the relation into fragments. Large number of database fragments is invented in this method, causing a high interacting cost for transmitting and processing the fragments.

B. Clustering Websites:

Clustering websites help to reduce communication cost \& the enhance the performance of the web database system. Clustering website is an open problem \& optimal solution to this problem is NP-complete. This effective method has essential role in minimizing processing of data during transformation and obtain process.

The clustering technique identifies groups of network sites in huge web database systems and discover data distributions in better way. This techniques is supposed to be an efficient method that has major role in reducing the amount of transferred and accessed data during processing database transactions system. When large number of sites in web database system increase to large scale, the problem of supporting high system performance with consistency and availability constraints become critical.

\section{Data Allocation:}

Data allocation helps in distributing the database fragments among the clusters \& their respective sites in the distributed database system. Data allocation is the technique which is used for allocating fragments to the websites clusters. In this approach addresses of assignment of networking nodes to each of the fragment in it. An efficiency of data allocation technique is measured in term of responsive time. The main purpose is to reduce the communication cost for data access, re-fragmentation of data and re-allocation management.

Data allocation is method of distributing the database fragments among the clusters and various sites in distributed database systems. This process advise that assignment of network node to each fragments in it. NP-complete problem is finding by an optimal data allocation. In this method distributing data fragments among database websites increase database system performance by decreasing data transferred and accessed during execution, reducing storage overhead, and improve availability and reliability. 
Vol. 6, Issue 4, April 2017

\section{PROPOSED SYSTEM OF IFCA}

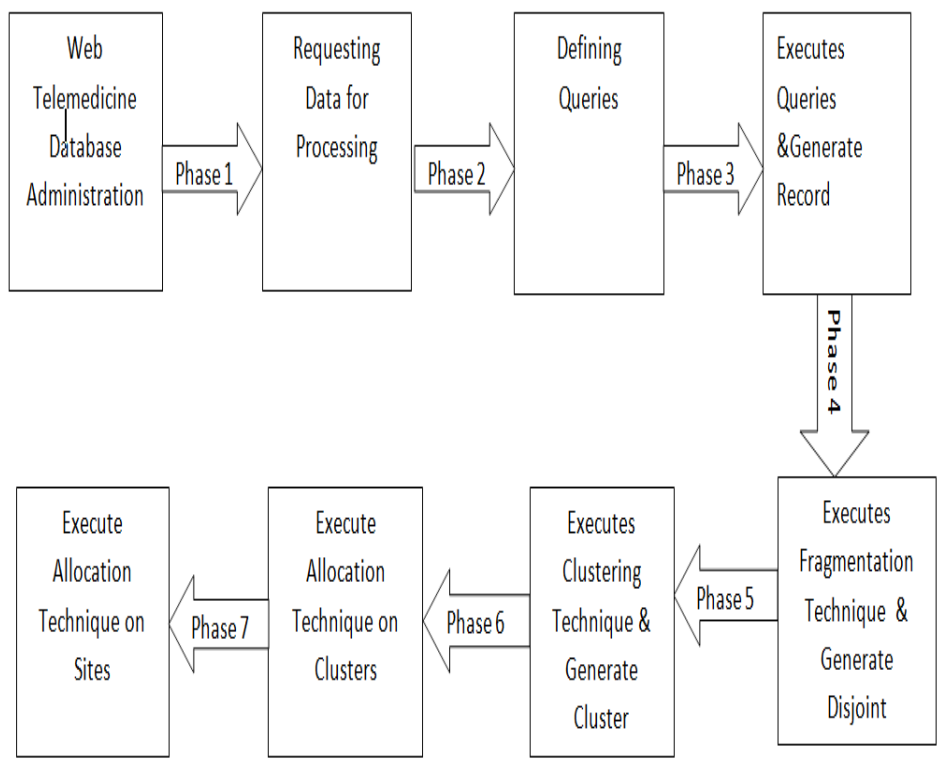

Fig. Proposed System

This scenario is called as Integrated-Fragmentation-Clustering-Allocation approach. Infig. Proposed System, the Telemedicine Integrated-Fragmentation-Clustering-Allocation (IFCA) architecture has six phased. In the first stage, generally data is requested start from the telemedicine database system sites. In second phase, two queries are defined for all the requested data which will be executed on the database system to generate various data set in third phase. After that in fourth phase, using the fragmentation technique execute data set which produces the disjoint fragments in it. In fifth phase, by using clustering technique and generate many numbers of clusters in it. The purpose of this clustering is to reduce the cost for data allocation whenever it is in use. In sixth phase by using allocation technique we execute clusters. At the end in seventh phase, by using allocation and distribution technique on the clusters and sites respectively. Database administration is only responsible for recovering any sites failure in web telemedicine database system. Therefore it is very important in telemedicine database management system to improve medical care.

\section{ARCHITECTURAL DESIGN}

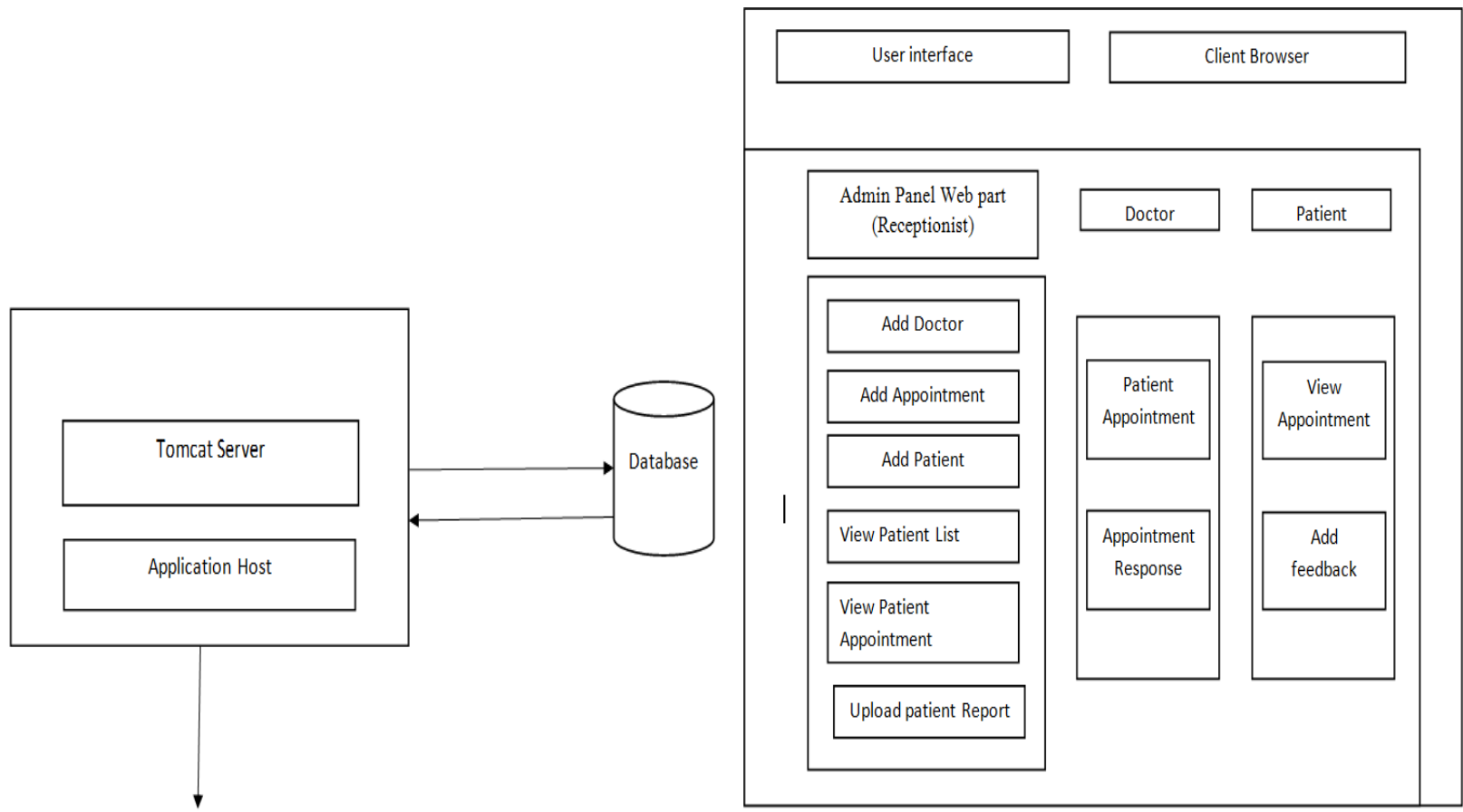


- $\quad$ To detect Diseases.

- Show All Appointments.

- All Appointment history.

\section{CONCLUSION}

In this work, we proposed a new approach to enhance web based telemedicine database management system performance. This work is introduced three fold approach. This paper combines these three approaches like data fragmentation, web-site clustering and data allocation. The main aim of this system is to accomplish the system throughput in terms of reliability, availability, and data consistency. This system improves communication and minimize communication cost.

Doctors need not worry about managing their appointment. This application will help to optimize the work of patient and doctor. The doctor is also able to view his day to day appointment list which makes it easier for him to plan his schedule. It's a complete doctor patient interaction application which is being automated for the ease of use.

\section{ACKNOWLEDGMENT}

We would like to express my deepest appreciation to all who provided me the possibility to complete this paper. A special gratitude we give to our project guide Mr. Rohit Bamane, whose contribution in stimulating suggestions and encouragement, helped me in writing this paper.

\section{REFERENCES}

[1] Hafez Fouad, 'Web-Based Database Management System for telemedicine System', Microelectronics Dept., Electronics Research Institute, Cairo, Egypt,2014

[2] Ismail Hababeh, Issa Khalil, and Abdallah Khrieshah, 'Designing High Performance Web-based Computing Service to Enhance Telemedicine Database Management System',2015.

[3] D. A. Vidhate1, Ige Pranita2, Kothari Pooja3, Kshatriya Pooja4, 'Executing Web-Based Computing Services To Achieve Performance And Help Telemedicine Database Management System', 2016.

[4] Hafez Fouad ,'Web-base Medical Informatics to Support Telemedicine System', Microelectonics Dept., Electonics Research Institute, Cairo, Egypt, 2014.

[5] Lepakshi Goude , 'Achieving an Availability, Elasticity and Reliability of the Data Access in Cloud Computing', Science and Technologies, 2011.

[6] S. Jagannatha, M. Mrunalini, T. Kumar, and K. Kanth ,'Using Mixed Fragmentation in Distributed Database Using UML 2.0,' IPCSIT, V. 2, pp. 190-194, 2011.

[7] http://en.wikipedia.org/wiki/MongoDB, Nov. 2013. 